\title{
AN ITERATIVE PROCEDURE FOR CERTAIN NONLINEAR CIRCUITS ${ }^{1}$
}

\author{
WILLIAM J. SWARTZ
}

1. Introduction. It is well known that if a coil of inductance $L$, a resistor of resistance $R$, and a condenser of capacitance $C$ are connected in series with an external electromotive force $E(t)$, the resulting flow of current $x$ is determinable by elementary means. To be considered here is the nonlinear problem arising from the replacement of the linear resistor by a nonlinear resistor having an associated potential drop $h(x)$ where $h^{\prime}(x)$ is bounded between positive constants. Such a resistor has been termed quasi-linear by Duffin [1]. Thus we consider the equation

$$
L \frac{d^{2} x}{d t^{2}}+\frac{d h(x)}{d t}+\frac{x}{C}=E^{\prime}(t) .
$$

We assume that $h^{\prime}(x)$ is continuous and that $0<a \leqq h^{\prime}(x) \leqq b . E(t)$ is assumed real, periodic and absolutely continuous with $E^{\prime}(t)$ belonging to $L_{2}$, the class of Lebesgue measurable and integrable square functions.

Duffin [1] has proved the existence of a periodic solution of (1) and others have treated even more general types of nonlinearity in a second order equation. The role of this paper then is to give a proof which is different as well as constructive.

We shall employ some of the well known properties of Hilbert space and Fourier series. Unless otherwise noted all sums on $n$ are from minus infinity to plus infinity. For two functions $x$ and $y$ the inner product is $(x, y)=(2 \pi)^{-1} \int_{0}^{2 \pi} x \bar{y} d t$ where $\bar{y}$ is the conjugate of $y$. The norm is given by $\|x\|^{2}=(x, x)$. As already indicated $L_{2}(0,2 \pi)$ (briefly $L_{2}$ hereafter) will denote the Hilbert space of functions Lebesgue measurable and integrable square in $0 \leqq t \leqq 2 \pi$. For an infinite-tuple $\mathrm{x}$ the norm is to be given by $\|\mathrm{x}\|{ }^{2}=\sum_{n}\left|x_{n}\right|^{2}$. If $\mathrm{x}$ refers to the F. C. (Fourier coefficients) of $x$ then $\|x\|=\|x\|$. In fact, use will be made of the correspondence between elements of $L_{2}$ and $H$, the Hilbert space of sequences $\left\{x_{n}\right\}$ such that $\sum_{n}\left|x_{n}\right|^{2}$ converges [2, p. 15]. If $\left\|x-x_{n}\right\| \rightarrow 0$ as $n \rightarrow \infty$, the sequence $\left\{x_{n}\right\}$ is said to converge in mean to $x$. We shall use the symbol $\doteq$ to refer to this normwise con-

Received by the editors January 23, 1958.

1 This paper contains part of a doctoral dissertation written at Iowa State College under the direction of Professor C. E. Langenhop. 
vergence. Ordinary or pointwise convergence will be indicated by the usual equality.

We shall give several lemmas in order that the following arguments may be self contained. These results are known or easily proven by standard procedures.

Lemma 1. If $\sum_{n}\left|i n x_{n}\right|^{2}$ converges then the $L_{2}$ function $y(t) \doteq$ $\sum_{n} i n x_{n} e^{i n t}$ is the derivative of $x(t)=\sum_{n} x_{n} e^{i n t}$ almost everywhere. Furthermore the latter series is absolutely and uniformly convergent and $x(t)$ is absolutely continuous.

Lemma 2. Let $x_{k}(t) \doteq \sum_{n} x_{n}^{(k)} e^{i n t}$ and $x_{k}=\left(x_{0}^{(k)}, x_{-1}^{(k)}, x_{1}^{(k)}, \cdots\right)$. Let $\mathbf{x}=\left(x_{0}, x_{-1}, x_{1}, \cdots\right)$ be such that $\left\|\mathbf{x}-\mathbf{x}_{k}\right\| \rightarrow 0$ as $k \rightarrow \infty$. Then there exists an $x(t) \in L_{2}$ such that $\left\|x(t)-x_{k}(t)\right\| \rightarrow 0$ as $k \rightarrow \infty$ and $x(t) \doteq$ $\sum_{n} x_{n} e^{i n t}$.

Lemma 3. Given the differential equation $d^{2} x / d t^{2}+\alpha(d x / d t)+\beta x$ $=f(t)$ where $\alpha, \beta$ are constants, $f(t+2 \pi)=f(t)$, and $f(t) \in L_{2}$. Let $L(n)$ $=\beta-n^{2}+i n \alpha$ be nonzero for all integers $n$. Let $x_{n}(t)=A_{n} / L(n) e^{i n t}$ where the $A_{n}$ are the F.C. of $f(t)$. Then the series $\sum_{n} x_{n}(t)$ converges uniformly over the infinite interval to a function $x(t)$, periodic of period $2 \pi$, such that $x^{\prime}(t)$ is absolutely continuous and which satisfies the given differential almost everywhere.

2. Description of the iteration. Before proceeding with a description of the iteration technique we rewrite (1) in a somewhat different form. We change the scale of the independent variable so that the forcing term shall have period $2 \pi$. Substituting $h(x)=b f(x)$ gives $0<p \leqq f^{\prime}(x) \leqq 1$ where $p=a / b$. Equation (1) becomes

$$
\frac{d^{2} x}{d t^{2}}+\alpha \frac{d f(x)}{d t}+\beta x=E^{\prime}(t)
$$

where $\alpha=b / L$ and $\beta=1 / L C$. This equation may be written

$$
\frac{d^{2} x}{d t^{2}}+\alpha \frac{d x}{d t}+\beta x=\frac{d}{d t}\left[E(t)-\alpha(p-1)\left\{\frac{x-f(x)}{1-p}\right\}\right] .
$$

The restriction on $f^{\prime}(x)$ may be written

$$
0 \leqq \frac{1-f^{\prime}(x)}{1-p} \leqq 1
$$

and if we let

$$
g(x)=\frac{x-f(x)}{1-p},
$$


then $0 \leqq g^{\prime}(x) \leqq 1$. Also let $\gamma=\alpha(p-1)$. Then, since $\alpha \neq 0,|\gamma|<|\alpha|$. The differential equation and accompanying restrictions now become:

$$
\frac{d^{2} x}{d t^{2}}+\alpha \frac{d x}{d t}+\beta x=\frac{d}{d t}[E(t)-\gamma g(x)]
$$

where (a) $\alpha, \beta \neq 0$ and $|\gamma|<|\alpha|$,

(b) $g^{\prime}(x)$ is continuous and $0 \leqq g^{\prime}(x) \leqq 1$,

(c) $E(t)$ is periodic (of period $2 \pi$ ), real, absolutely continuous and $E^{\prime}(t) \in L_{2}$.

The solution of (4) is to be approximated by a sequence of functions $\left\{x_{k}(t)\right\}$ determined as follows: $x_{0}(t)$ is the periodic solution of the linear differential equation resulting from the deletion of the nonlinear term $g(x)$ from the right member of (4). Now $x_{0}(t)$ is substituted into the right member of $(4)$ and $x_{1}(t)$ determined as the periodic solution of this linear equation. In general, the $(k+1)$ st approximation is the periodic solution of

$$
\frac{d^{2} x}{d t^{2}}+\alpha \frac{d x}{d t}+\beta x=\frac{d}{d t}\left[E(t)-\gamma g\left(x_{k}(t)\right)\right] .
$$

In order to regard this iteration from a different viewpoint, suppose that both $x_{k}(t)$ and $g\left(x_{k}(t)\right)$ have the following Fourier series representations:

$$
\begin{array}{rlrl}
x_{k}(t) & =\sum_{n} x_{n}^{(k)} e^{i n t} & & (k=0,1,2, \cdots), \\
g\left(x_{k}(t)\right) & =\sum_{n} g_{n}^{(k)} e^{i n t}, & & \\
g_{n}^{(k)} & =\left(g\left(x_{k}(t)\right), e^{i n t}\right) & (k=0,1,2, \cdots),
\end{array}
$$

$E(t)$, being absolutely continuous, is of bounded variation and has the representation

$$
E(t)=\sum_{n} E_{n} e^{i n t} .
$$

If the series expressions for $x_{k+1}(t), g\left(x_{k}(t)\right)$ and $E(t)$ are formally substituted into (5) and coefficients of corresponding harmonics are equated, the result is

$$
x_{n}^{(k+1)}=\frac{i n\left(E_{n}-\gamma g_{n}^{(k)}\right)}{\beta-n^{2}+i n \alpha} \quad(n=0, \mp 1, \mp 2, \cdots) .
$$

Now the iteration may be carried out in the following way. As the beginning approximation take 


$$
x_{n}^{(0)}=\frac{i n E_{n}}{\beta-n^{2}+i n \alpha} \quad(n=0, \mp 1, \mp 2, \cdots)
$$

(for these are the F.C. given by (9) with $g$ deleted). Then (7) with $k=0$ is used to calculate the $g_{n}^{(0)}$. (9) is now used to calculate the F.C. $x_{n}^{(1)}$ of $x_{1}(t)$. A repetition of this procedure gives successive approximations.

Since the infinite-tuple of F.C. of $x_{k}(t)$ belongs to the Hilbert space $H$, this iteration may be regarded as a successive transformation in the space $H$. Thus, if the infinite-tuple $x_{k}$ is defined by $x_{k}=\left(x_{0}^{(k)}\right.$, $\left.x_{-1}^{(k)}, x_{1}^{(k)}, x_{-2}^{(k)}, \cdots\right)$, the transformation $F\left(x_{k}\right)$ is given in accordance with (9) by

$$
\begin{aligned}
F\left(\mathbf{x}_{k}\right)=\left(0, \frac{-i\left(E_{-1}-\gamma g_{-1}^{(k)}\right)}{\beta-1-\alpha i}, \frac{i\left(E_{1}-\gamma g_{1}^{(k)}\right)}{\beta-1+\alpha i}\right. \\
\left.\frac{-2 i\left(E_{-2}-\gamma g_{-2}^{(k)}\right)}{\beta-4-2 \alpha i}, \ldots\right) .
\end{aligned}
$$

Then the iteration is described by the relation

$$
\mathbf{x}_{k+1}=F\left(\mathbf{x}_{k}\right) \quad(k=0,1,2, \cdots) .
$$

In the following we shall justify the heuristic procedures of this section.

3. Proof of convergence. The existence of the iterates $x_{k}(t)$ is argued by induction. According to Lemma 3 a periodic $x_{0}(t)$ exists. Suppose that $x_{k}(t)$ is periodic and such that $x_{k}^{\prime}(t)$ is absolutely continuous. $g^{\prime}\left(x_{k}(t)\right)$ being continuous is measurable as well as bounded and belongs to $L_{2}$. Thus the time derivative of $g\left(x_{k}(t)\right)$ belongs to $L_{2}$ and so also does the right member of (5). Now by Lemma 3, there exists a periodic function $x_{k+1}(t)$ such that $x_{k+1}^{\prime}(t)$ is absolutely continuous and which satisfies (5) almost everywhere. The F.C. of the right member of (5) are in $\left(E_{n}-\gamma g_{n}^{(\boldsymbol{k})}\right)$ since $g$ as well as $E(t)$ is absolutely continuous [3, p. 23]. Hence, by Lemma 3 , the F.C. of $x_{k+1}(t)$ are given by $(9)$. We note also that since it has a continuous derivative, $g\left(x_{k}(t)\right)$, as well as $x_{k}(t)$, is absolutely continuous and therefore the series representations (6) and (7) are justified. It is also easily argued by induction that all $x_{k}(t)$ are real.

Let $x_{r}(t)$ and $x_{s}(t)$ be any two real, absolutely continuous functions whatsoever with $x_{r}^{\prime}(t)$ and $x_{s}^{\prime}(t)$ belonging to $L_{2}$. Substitute these functions into the right member of (4). Then periodic solutions, say 
$y_{r}(t)$ and $y_{s}(t)$ respectively, exist whose F.C. are given by

$$
y_{n}^{(r)}=\frac{i n\left(E_{n}-\gamma g_{n}^{(r)}\right)}{\beta-n^{2}+i n \alpha} \text { and } y_{n}^{(s)}=\frac{i n\left(E_{n}-\gamma g_{n}^{(s)}\right)}{\beta-n^{2}+i n \alpha} .
$$

According to (10) these relations may be written $y_{r}=F\left(x_{r}\right)$ and $y_{s}=F\left(x_{s}\right)$.

In view of the bounds on $g^{\prime}$, the law of the mean gives

$$
\left|g\left(x_{r}\right)-g\left(x_{s}\right)\right|^{2} \leqq\left(x_{r}-x_{s}\right)^{2} .
$$

Therefore

$$
\left\|g\left(x_{r}\right)-g\left(x_{8}\right)\right\|^{2} \leqq\left\|x_{r}-x_{8}\right\|^{2} .
$$

From (10) one has

$$
\begin{aligned}
\left\|F\left(\mathbf{x}_{r}\right)-F\left(\mathrm{x}_{s}\right)\right\|^{2} & =\sum_{n} \frac{n^{2} \gamma^{2}\left|g_{n}^{(r)}-g_{n}^{(s)}\right|^{2}}{\left(\beta-n^{2}\right)^{2}+n^{2} \alpha^{2}} \\
& =\gamma^{2} \sum_{n} \frac{\left|g_{n}^{(r)}-g_{n}^{(s)}\right|^{2}}{(\beta / n-n)^{2}+\alpha^{2}} \quad(n \neq 0) \\
& \leqq \gamma^{2} / \alpha^{2} \sum_{n}\left|g_{n}^{(r)}-g_{n}^{(s)}\right|^{2} \\
& =\gamma^{2} / \alpha^{2}\left\|g\left(x_{r}\right)-g\left(x_{s}\right)\right\|^{2} .
\end{aligned}
$$

Together with (12) this result gives

$$
\left\|F\left(\mathbf{x}_{r}\right)-F\left(\mathbf{x}_{s}\right)\right\| \leqq|\gamma / \alpha|\left\|x_{r}-x_{s}\right\|=|\gamma / \alpha|\left\|\mathbf{x}_{r}-\mathbf{x}_{s}\right\| .
$$

From (11), $\left\|x_{2}-x_{1}\right\|=\left\|F\left(x_{1}\right)-F\left(x_{0}\right)\right\|$ is obtained and then (13) gives $\left\|x_{2}-x_{1}|| \leqq|\gamma / \alpha|\right\| x_{1}-x_{0}||$. Now assume that

$$
\left\|\mathbf{x}_{k+1}-\mathbf{x}_{k}|| \leqq|\gamma / \alpha| k \mid \mathbf{x}_{1}-\mathbf{x}_{0}\right\| \text {. }
$$

Again (11) and (13) give $\left\|x_{k+2}-x_{k+1}|| \leqq|\gamma / \alpha|\right\| x_{k+1}-x_{k} \|$. But in view of the assumption (14) this is $\left\|x_{k+2}-x_{k+1}|| \leqq|\gamma / \alpha|^{k+1}\right\| x_{1}-x_{0} \|$. Hence by induction on $k,(14)$ is true for all positive integers $k$. Consider now $\left\|x_{k+p}-x_{k}\right\|$ where $p$ is any positive integer. Using (14) and letting $K=\left\|x_{1}-x_{0}\right\|$ gives

$$
\begin{aligned}
\left\|\mathbf{x}_{k+p}-\mathbf{x}_{k}\right\| & \leqq\left\|\mathbf{x}_{k+p}-\mathbf{x}_{k+p-1}\right\|+\left\|\mathbf{x}_{k+p-1}-\mathbf{x}_{k+p-2}\right\|+\cdots+\left\|\mathbf{x}_{k+1}-\mathbf{x}_{k}\right\| \\
& \leqq K|\gamma / \alpha|^{k+p-1}+K|\gamma / \alpha|^{k+p-2}+\cdots+K|\gamma / \alpha|^{k} \\
& \leqq \frac{K}{1-|\gamma / \alpha|}|\gamma / \alpha|^{k} .
\end{aligned}
$$


Since $|\gamma|<|\alpha|,\left\|x_{k+p}-x_{k}\right\| \rightarrow 0$ as $k \rightarrow \infty$; that is $\left\{x_{k}\right\}$ is a Cauchy sequence in $H$. Since $H$ is complete there exists an $x \in H$ such that $\left\|\boldsymbol{x}-\mathbf{x}_{k}\right\| \rightarrow 0$ as $k \rightarrow \infty$. Lemma 2 gives $x(t) \doteq \lim _{k \rightarrow \infty} x_{k}(t)$ in the space $L_{2}$ where F.C. of $x(t)$ are given by $\mathrm{x}$. That this convergence is indeed pointwise will be noted in the next section.

4. Verification of the solution. We shall show that the limit function $x(t)$ satisfies (4). Equation (9) yields

$$
\begin{aligned}
\left|i n x_{n}^{(k+p)}-i n x_{n}^{(k)}\right|^{2} & =\left|\frac{-n^{2} \gamma}{\beta-n^{2}+i n \alpha}\right|^{2}\left|g_{n}^{(k+p-1)}-g_{n}^{(k-1)}\right|^{2} \\
& \leqq M\left|g_{n}^{(k+p-1)}-g_{n}^{(k-1)}\right|^{2}
\end{aligned}
$$

where $M$ is a constant independent of $n$. Summing on $n$ and using (12) gives

$$
\begin{aligned}
\sum_{n}\left|i n x_{n}^{(k+p)}-i n x_{n}^{(k)}\right|^{2} & \leqq M\left\|g\left(x_{k+p-1}(t)\right)-g\left(x_{k-1}(t)\right)\right\|^{2} \\
& \leqq M\left\|x_{k+p-1}(t)-x_{k-1}(t)\right\|^{2}
\end{aligned}
$$

and establishes the convergence of the series on the left. Hence, according to Lemma 1 , this series is $\left\|x_{k+p}^{\prime}(t)-x_{k}^{\prime}(t)\right\|^{2}$. It follows, since $\left\{x_{k}(t)\right\}$ is a Cauchy sequence, that $\left\{x_{k}^{\prime}(t)\right\}$ is also a Cauchy sequence in $L_{2}$ and therefore has a limit, say $u(t)$, belonging to $L_{2}$. For $0 \leqq t \leqq 2 \pi$ one may write

$$
\begin{aligned}
\| \int_{0}^{t} x_{k}^{\prime}(s) d s- & \int_{0}^{t} u(s) d s \|^{2}=1 / 2 \pi \int_{0}^{2 \pi}\left[\int_{0}^{t}\left(x_{k}^{\prime}(s)-u(s)\right) d s\right]^{2} d t \\
& \leqq 1 / 2 \pi \int_{0}^{2 \pi}\left[\int_{0}^{t} d s\right]\left[\int_{0}^{t}\left(x_{k}^{\prime}(s)-u(s)\right)^{2} d s\right] d t \\
& \leqq \int_{0}^{2 \pi} \int_{0}^{2 \pi}\left(x_{k}^{\prime}(s)-u(s)\right)^{2} d s d t=4 \pi^{2}\left\|x_{k}^{\prime}(t)-u(t)\right\|^{2} .
\end{aligned}
$$

Thus

$$
\lim _{k \rightarrow \infty} \int_{0}^{t} x_{k}^{\prime}(s) d s \doteq \int_{0}^{t} u(s) d s .
$$

The $x_{k}^{\prime}(s)$, and hence $u(s)$, have zero average over $0 \leqq t \leqq 2 \pi$. The right member is then periodic as well as absolutely continuous and may therefore be written as $\sum_{n} f_{n} e^{i n t}$ where $\sum_{n}\left|n f_{n}\right|^{2}$ converges [3, p. 23]. Furthermore $x_{k}(t)$ is absolutely continuous so the left member is $\lim _{k \rightarrow \infty}\left(x_{k}(t)-x_{k}(0)\right)$. Now let $C=\lim _{k \rightarrow \infty} x_{k}(0)$ and write 


$$
\begin{aligned}
\left\|x(t)-\left(C+\sum_{-m}^{m} f_{n} e^{i n t}\right)\right\| & \\
\leqq & \left\|x(t)-x_{k}(t)\right\|+\left\|x_{k}(t)-x_{k}(0)-\int_{0}^{t} u(s) d s\right\| \\
& \quad+\left\|x_{k}(0)-C\right\|+\left\|\int_{0}^{t} u(s) d s-\sum_{-m}^{m} f_{n} e^{i n t}\right\| .
\end{aligned}
$$

As $m$ and $k$ approach infinity the norms in the right member approach zero and we have $x(t) \doteq C+\sum_{n} f_{n} e^{i n t}$. Since the F.C. of $x(t)$ have been shown to be $x_{n}$, one now has $\sum_{n} \mid$ in $\left.x_{n}\right|^{2}$ convergent and Lemma 1 gives $v(t) \doteq \sum_{n}$ in $x_{n} e^{\text {int }}$ with $x^{\prime}(t)=v(t)$ almost everywhere where $v(t)$, and hence $x^{\prime}(t)$, belong to $L_{2}$. It is also easily deduced that $v(t)$ and $x^{\prime}(t)$ are equivalent to $u(t)$.

Substitute $x(t)$ into the right member of (4). Since $x^{\prime}(t) \in L_{2}$ the right member belongs to $L_{2}$, and according to Lemma 3 a periodic solution, say $x^{*}(t)$, exists. Thus

$$
\frac{d^{2} x^{*}(t)}{d t^{2}}+\alpha \frac{d x^{*}(t)}{d t}+\beta x^{*}(t) \equiv \frac{d}{d t}[E(t)-\gamma g(x(t))]
$$

almost everywhere, and consequently, $x^{*}=F(x)$. Now (13) may be applied to give $\left\|F\left(x_{k}\right)-F(x)\right\| \leqq|\gamma / \alpha|\left\|x_{k}-x\right\|$. But $F\left(x_{k}\right)=x_{k+1}$ and $\left\|x_{k}-x\right\| \rightarrow 0$ as $k \rightarrow \infty$. Thus $\lim _{k \rightarrow \infty} x_{k+1}=F(x)$ or $x=F(x)$. Hence $\mathrm{x}^{*}=\mathrm{x}$ or $x^{*}(t)=x(t)$ almost everywhere. Replacement of $x^{*}(t)$ by $x(t)$ in the identity above verifies that $x(t)$ is a solution of (4) almost everywhere.

To show that the convergence is pointwise consider (6) and the relation $x(t)=\sum_{n} x_{n} e^{i n t}$ which is valid since $x(t)$ is absolutely continuous. Use of the Schwarz inequality and the convergence of $\sum_{n}\left|n x_{n}\right|^{2}$ and $\sum_{n}\left|n x_{n}^{(k)}\right|^{2}$ shows that these series are absolutely and uniformly convergent. One may now write

$$
\begin{aligned}
\left|x(t)-x_{k}(t)\right| & =\left|\sum_{n}\left(x_{n}-x_{n}^{(k)}\right) e^{i n t}\right| \\
& \leqq \sum_{n}\left|x_{n}-x_{n}^{(k)}\right| \\
& \leqq\left(\sum_{n} 1 / n^{2}\right)^{1 / 2}\left(\sum_{n}\left|n x_{n}-n x_{n}^{(k)}\right|^{2}\right)^{1 / 2} \\
& =\left(\sum_{n} 1 / n^{2}\right)^{1 / 2}\left\|x^{\prime}(t)-x_{k}^{\prime}(t)\right\|
\end{aligned}
$$

where the sums need not be taken over $n=0$ since $x_{0}^{(k)}=0$ for all $k$. 
The norm on the right approaches zero as $k \rightarrow \infty$. Furthermore, since it is independent of $t$, the convergence of $x_{k}(t)$ to $x(t)$ is uniform as well as pointwise.

5. Remarks. Equation (2) was written in the form (3) by adding a linear term to both members. It is interesting to note the consequences if this tactic is not employed. If (2) is attacked directly one must assume $\beta \neq n^{2}$ for all integers $n$ so existence of the iterates is not destroyed by the presence of resonance type terms. Furthermore, even with this assumption, the proof of convergence demands a further restriction on the parameters $\alpha$ and $\beta$.

The author has applied these techniques to the matrix differential equation corresponding to a network containing quasi-linear resistors and has proven convergence subject to certain restrictions on the circuit parameters.

\section{REFERENCES}

1. R. J. Duffin, Nonlinear networks III, Bull. Amer. Math. Soc. vol. 55 (1949) pp. 119-129.

2. M. H. Stone, Linear transformations in Hilbert space, Amer. Math. Soc. Colloquium Publications, no. 15, 1932.

3. G. H. Hardy and W. IV. Rogosinski, Fourier series, Cambridge Tracts No. 38, Cambridge University Press, 1944.

Iowa State College and Montana State College 